\title{
Effects of lateral-chain thiophene fluorination on morphology and charge transport of BDT-T based small molecule donors: A study with multiscale simulations
}

\author{
Chunlin $\mathrm{Xu},{ }^{\mathrm{a}}$ Chuang Yao, ${ }^{* \mathrm{~b}}$ and Shaohui Zheng ${ }^{* \mathrm{a}}$ \\ ${ }^{a}$ Chongqing Key Laboratory for Advanced Materials and Technologies of Clean Energies, \\ School of Materials and Energy, Southwest University, Chongqing 400715, P.R. China; \\ ${ }^{b}$ Key Laboratory of Extraordinary Bond Engineering and Advance Materials Technology \\ (EBEAM) of Chongqing, \\ School of Materials Science and Engineering, Yangtze Normal University, Chongqing 408100, \\ P.R. China; \\ *Email: yaochuang@yznu.cn, shaohuizheng@swu.edu.cn
}

\begin{abstract}
Thiophene substituted benzo[1,2-b:4,5-b' ]dithiophene (BDT-T) is widely used as the building block of promising donor materials in organic solar cells (OSCs). Fluorination on the lateral-chain thiophenes of BDT-T is a considerable strategy to further improve the performance of BDT-T based small molecule (SM) donors. However, how lateral-chain thiophene fluorination affects the morphology and hole mobility $\left(\mu_{h}\right)$ of SM donors has not been well understood. In the present work, we systematically study the effects of the positions and numbers of fluorinations on the morphology and $\mu_{h}$ of a promising SM donor named DRTB-T (referred as 0F) with multiple fluorinations via multiscale simulations. We find the crystallinity is weakened because of fluorination. The face-on configurations are dominated in $0 \mathrm{~F}$ and 1F-substituted molecules. In contrast, the edge-on configurations are dominated in 2F-substituted molecules. The trend of $\mu_{h} \mathrm{~s}$ in these fluorinated molecules is consistent with that of the proportions of face-on configurations, and matches well with available experimental results. We find that functionalizing the lateral-chain thiophenes of side BDT-Ts (1F-side) or all BDT-Ts (1F-all) with only one F atom are efficient to fine-tune the morphologies and $\mu_{h}$ of DRTB-T based SM donors simultaneously.
\end{abstract}

Keywords: Organic solar cells; Fluorination; Morphology; Hole mobility; Molecular dynamics. 


\section{Introduction}

Exciting progresses have been achieved in the research field of organic solar cells (OSCs) in recent years, and the power conversion efficiency (PCE) has been up to $18 \%$ by combining polymer donors and non-fullerene small molecule (SM) acceptors. ${ }^{1-4}$ Compared to polymers, SMs have well-defined structure, high purity, and good reproducibility for mass production. ${ }^{5-10}$ Hence, all SM OSCs based on both SM donors and acceptors have caught considerable attentions. ${ }^{5-10}$ For SM acceptors, until five years ago, the most popular acceptors in OSCs are fullerene and their derivatives because they possess strong electron affinity and high electron mobility. ${ }^{11,12}$ However, fullerene materials have intrinsic disadvantages such as weak and narrow photo absorption, poor adjustability of energy levels, and easy aggregation, which greatly limit the development of fullerene-based OSCs. Therefore, researchers developed non-fullerene SM acceptors, especially the fused-ring electron acceptors (FREAs) such as Y6 and ITIC, which show advantages such as tunable energy levels, near-infrared absorption, and low energy loss. ${ }^{13-16}$ Thus, all-small-molecule (ASM) OSCs based on both SM donors and non-fullerene SM acceptors may be promising candidates of new-generation high performance OSCs. ${ }^{8,10,17-22}$

To date, the highest PCE of binary ASM OSCs has reached $15.3 \%,{ }^{19,23}$ but it is still lower than the polymer-based OSCs $(\sim 18 \%) .{ }^{1-4}$ Since the potential of the best non-fullerene FREAs has been proved in the high efficiency polymer-based OSCs, for ASM OSCs, its main task now is to improve available SM donor materials or find new promising SM donors. It has been always hard to find a new revolutionary material if going over the history of OSC. Hence, keeping improving available SM donors is an efficient and useful way. So far, the most popular building block of promising donor materials including $\mathrm{SMs}$ and polymers is benzo[1,2-b:4,5-b' ]dithiophene (BDT). Owing to its rigid and planar conjugated structure, the donor with a BDT unit as the core of backbone generally has the tunable molecular energy levels and energy gaps as well as high hole mobilities. ${ }^{24,25}$ 
To further improve the donors with BDT units, researchers functionalized BDT with two lateral-chain thiophenes to improve charge delocalization from one to two dimensions and introduce more active sites for molecular modification. ${ }^{25}$ Actually, most of efficient donor materials have thiophene substituted BDT (BDT-T) as a core building block, instead of just BDT. On the other hand, as one of the simplest and efficient strategy of molecular engineering, fluorination can downshift molecular energy level, enhance intermolecular interaction, and tune blend morphology of active layer. ${ }^{26-28}$ Hence, fluorinations on the lateral-chain thiophenes of BDT-T have been tested, and several impressive experimental progresses have been made. To date, the fluorinated BDT-T can be found in the best polymer donors such as D18 ${ }^{1}$ and PM6. ${ }^{29}$ Also, it has been introduced into many promising SM donors. For instance, Zhu et al. ${ }^{30}$ synthesized a new donor material named BSFTR by using fluorinated BDT-T as electron-donating moiety; the PCE of the OSC based on BSFTR (donor: D) and the well-known non-fullerene acceptor Y6 (acceptor: A) reached 13.7\%. ${ }^{14,17}$ By carefully controlling the number of fluorination on one lateral-chain thiophene of BDT-T of a SM donor DCAO3TBDTT, Ge et al. synthesized two new molecules named BTEC-1F and BTEC-2F; they found that the PCEs of SM OSCs increased with the number of substituted $\mathrm{F}$ atoms on BDT-T unit, that is, BTEC-2F $>$ BTEC-1F > DCAO3TBDTT, and achieved a PCE up to $13.34 \% .{ }^{18}$ Besides, Yang et al. designed an asymmetric C-shaped SM donor named TBD-S4 with a fluorinated BDT-T unit. A remarkable PCE of $15.1 \%$ was reached when incorporating TBD-S4 with Y6. ${ }^{31}$ Very recently, Qiu et al. synthesized a new donor named DRTB-FT by fluorinating the lateral-chain thiophene of the center BDT-T unit of DRTB-T; interestingly, the highest PCE of DRTB-FT (D): F-2Cl (A) OSC (7.66\%) is much lower than that based on the prototype molecule DRTB-T (10.76\%). ${ }^{32,33}$ Clearly, fluorination on BDT-T core units of SM donors does not always increase the PCE of an OSC. Therefore, one question arises, that is, how fluorination on BDT-T unit affects the performance of a donor.

Until now, there are rare studies on the relations between lateral-chain thiophene fluorination of BDT-T core and the performance of BDT-T based SM donors. Especially, how lateral-chain thiophene fluorination affects morphology, which is 
crucial but hard to study by experiments, is still confusing. To shed some light on this issue, in this paper, we choose a promising SM donor named DRTB-T designed by Hou et al. ${ }^{7}$ as an example to study the effects of lateral-chain thiophene fluorination of BDT-T unit on morphology and hole mobility. DRTB-T contains three repeated BDT-Ts as donor unit, end-capping with two 2-thioxo-4-thiazolidinones (rhodanines) as acceptor units. The structure of DRTB-T provides us abundant active sites to consider different numbers and positions of fluorinations. In addition, the highest PCE of ASM OSCs based on DRTB-T donor is up to $11.24 \% .{ }^{34}$ It further motivates us to choose DRTB-T to perform this study. Hence, we design a serial of fluorinated DRTB-T derivatives by changing the positions and numbers of the lateral-chain thiophene fluorinations on the core BDT-T units, and comprehensively investigate the molecular stacking and hole mobility by means of multiscale simulations.

By functionalizing the lateral-chain thiophenes of three BDT-Ts of DRTB-T with fluorine atoms, we have modelled six molecules, namely: 1F-center, 1F-side, 1F-all, 2F-center, 2F-side, and 2F-all, as shown in Fig. 1. Here, "1" or "2" means the number of $\mathrm{F}$ atom substituted on each lateral-chain thiophene, and "center" or "side" denotes the center and both side positions of fluorinated thiophene ring respectively. Plus, "all" means that all lateral-chain thiophene rings of three BDT-T units are fluorinated. The prototype DRTB-T is referred as " $0 F$ " hereafter for consistency and clarity.

Since classical molecule dynamics (CMD) simulation has been successfully been applied for obtain the details of morphology of organic photovoltaic materials, ${ }^{35-38}$ we use it to investigate the molecular packing of DRTB-T and its fluorinated derivatives in amorphous forms. Then, quantum chemical calculations and kinetic Monte Carlo simulation $(\mathrm{KMC})^{39,40}$ are utilized to obtain the hole mobility in thin films of these materials.

The purposes of this project are two-fold:

1) To study the effects of the positions and numbers of fluorinations of the lateral-chain thiophenes of the core BDT-T units on the morphology.

2) To gain an insight about the relationship between fluorination and hole mobility. 

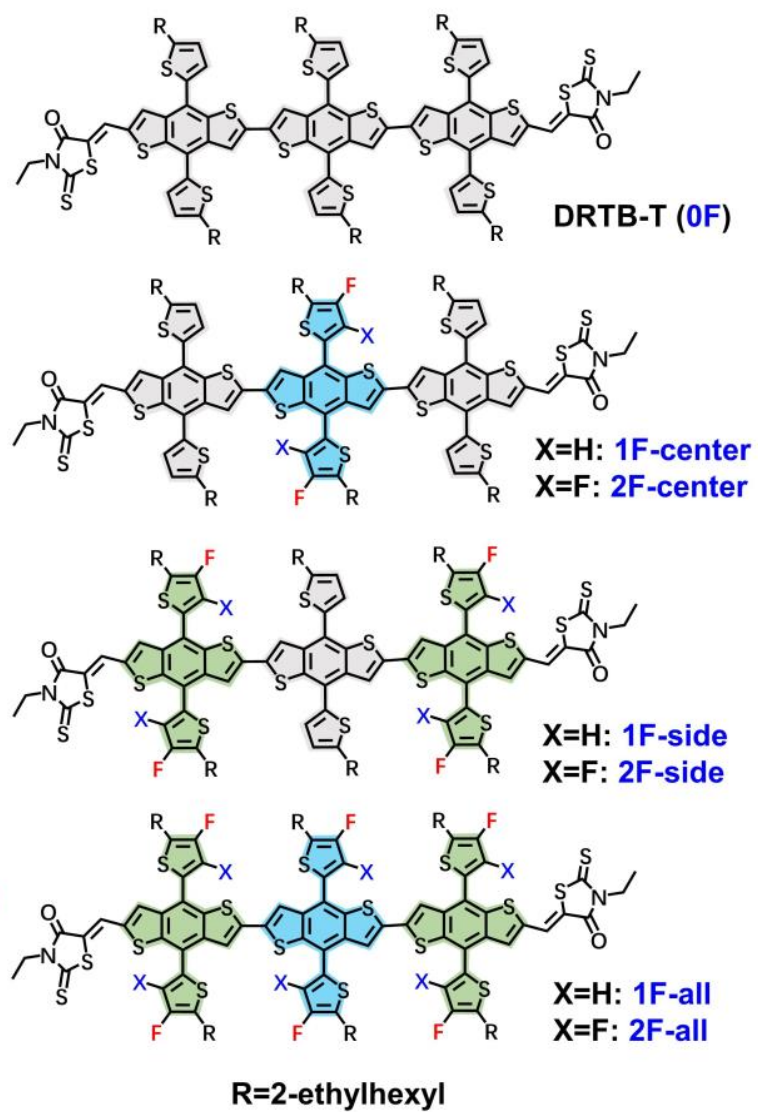

Fig. 1 Chemical structures of the modeled fluorinated DRTB-T molecules: 0F, 1F-center, 1F-side, 1F-all, 2F-center, 2F-side, and 2F-all.

\section{Computational details}

MD simulations. All CMD simulations were run with Gromacs 5.1.3 software package, ${ }^{41}$ and all quantum chemical calculations were performed with Gaussian 09 Rev. E01 package. ${ }^{42}$ The general AMBER force field $(\mathrm{GAFF})^{43,44}$ was used to accomplish CMD simulations, and the restrained electrostatic potential (RESP) charge was utilized for atomic charge. ${ }^{45}$ The combination of GAFF and RESP charges has been widely applied to simulate organic molecules. ${ }^{35-37}$ The RESP charge, frontier molecular orbital (FMO), and electrostatic potential (ESP) on the van del Waals surface were obtained by using Multiwfn Rev 3.7 program $^{46-48}$ based on B3LYP/6-31G** level of theory, which has been proved capable to well reproduce the experimental structure. ${ }^{49,50}$ The torsion potential parameters between side alkyl chain and backbone and between the BDT-T and ending group were re-parameterized via DFT calculations at B3LYP/6-31G** level, as shown in Supporting Information (SI) 
Fig. S1. To simulate the molecular packing in amorphous films, all CMD simulations were performed with following procedures: (1) 400 molecules were put into a large cubic box $(50 \mathrm{~nm} \times 50 \mathrm{~nm} \times 50 \mathrm{~nm})$ to make sure molecules orientate randomly; (2) 2000 steps of energy minimization based on the steepest descent method were run to remove undesired forces and structural distortion; (3) $5 \mathrm{~ns}$ simulation under $500 \mathrm{~K}$ and 100 bar were performed to close the gaps between molecules; (4) $10 \mathrm{~ns}$ simulation under $500 \mathrm{~K}$ and 1 bar, and then cooling down to $300 \mathrm{~K}$ gradually in $5 \mathrm{~ns}$ to simulate experimental thermal annealing (TA) process; (5) $20 \mathrm{~ns}$ simulation at 300 $\mathrm{K}$ and 1 bar to obtain equilibrated system. The size of final box is about $11 \mathrm{~nm}$, corresponding to the density around $1150 \mathrm{~kg} / \mathrm{m}^{3}$ which is in the reasonable density range $\left(1100 \sim 1150 \mathrm{~kg} / \mathrm{m}^{3}\right){ }^{36}$ All simulations were performed with $3 \mathrm{D}$ periodic boundary conditions (PBCs) by using a leap-frog integrator with a time step of $1.0 \mathrm{fs}$. The cut-off of van der Waals interactions, short-range electrostatic interactions, and the particle-mesh Ewald solver for long-rang electrostatic interactions was set to 1.2 $\mathrm{nm}$. The velocity rescaling thermostat ${ }^{51}$ and Berendsen barostat ${ }^{52}$ were used under the NPT ensemble, except for the last 10 ns simulation in step 5, in which the NoséHoover thermostat ${ }^{53,54}$ and Parrinello-Rahman barostat ${ }^{55}$ were utilized to gain better equilibrium systems. After the last $10 \mathrm{~ns}$ simulation, an additional 30 ps simulation was added, and we sampled every 30 fs for the statistics of the radical distribution functions (RDFs) and torsion angles.

Hole mobility calculations. We selected the hopping model ${ }^{56}$ to obtain hole mobility because the reorganization energies in our modelled systems are generally larger than electronic coupling. In the low-field limit, hole mobility can be obtained with the Einstein formula: ${ }^{57}$

$$
\mu=\frac{e D}{k_{B} T}
$$

Here, $\mathrm{D}$ is the charge diffusion coefficient, and it is defined as the ratio of the square of a displacement to diffusion time:

$$
D=\frac{1}{2 n} \lim _{t \rightarrow \infty} \frac{r(t)^{2}}{t}
$$

where ' $\mathrm{n}$ ' means n-dimensional system, and $r(t)$ is the diffusion displacement. Thus, 
we need calculate the slope of $r(t)^{2}$ and $t$ for hole mobility. A kinetic Monte Carlo simulation was run to mimic the hole hopping process in the amorphous films, which were obtained from the previous CMD simulations. For the details of the KMC simulation processes, please see our previous work. ${ }^{58,59}$ For all molecular pairs in KMC simulations, we treated the center-of-mass ( $\mathrm{COM})$ of each molecule as hopping point. Initially, one molecule $(i)$ was randomly selected in the CMD simulation box. Then, a hole transfers between the molecule $i$ and its adjacent molecule $(j)$. The probability of this charge hopping is defined as $P_{i j}=k_{i j} / \sum_{n} k_{i j}$, in which $k_{i j}$ is the hopping rate from the molecule $i$ to $j$ and can be obtained with the semi-classical Marcus formula: ${ }^{60}$

$$
k_{i j}=\frac{V_{i j}^{2}}{\hbar} \sqrt{\frac{\pi}{\lambda k_{B} T}} \exp \left[-\frac{\left(\Delta G_{i j}+\lambda\right)^{2}}{4 \lambda k_{B} T}\right]
$$

where $V_{i j}$ denotes the electronic coupling between the molecular pair (the $i$ th and $j$ th molecules), and was obtained with the Zerner's intermediate neglect of differential overlap (ZINDO) method; ${ }^{61} \hbar$ and $k_{B}$ are the reduced Planck and Boltzmann constants respectively; $T$ means the absolute temperature and set to $298.15 \mathrm{~K} ; \Delta G_{i j}$ represents the free energy difference of hole transfer between the $i$ th and $j$ th molecules, and was set to zero in our calculations; $\lambda$ denotes the reorganization energy and here we only considered the inner reorganization energy. We calculated the reorganization energy of hole transfer with following equation: ${ }^{62}$

$$
\lambda=\lambda_{0}+\lambda_{+}=\left(E_{0}^{*}-E_{0}\right)+\left(E_{+}^{*}-E_{+}\right)
$$

Here, $E_{0}^{*} / E_{0}$ is the total energy of the neutral molecule with the optimized geometry of charged/neutral molecule, correspondingly; $E_{+}^{*} / E_{+}$denotes the total energy of charged molecule with the optimized geometry of neutral/charged molecule, respectively. The hopping time and distance are defined as $1 / k_{i j}$ and the molecular $\operatorname{COM}(i)-\operatorname{COM}(j)$ distance. For each hopping step, if $\sum_{n=1}^{a-1} P_{i j}<r<\sum_{n=1}^{a} P_{i j}(r: \mathrm{a}$ random number between 0 and 1), then the hole is allowed to move from molecule $i$ to the $a$ th adjacent molecule $j$. This procedure was repeated by the next hopping step starting from this molecule. To end, the hopping coordinate and time were utilized to build the relationship between $r(t)^{2}$ and $t$ to obtain the charge diffusion 
coefficient.

\section{Results and discussion}

\subsection{Molecular structure of monomers}

We firstly discuss the effect of fluorination on the physical properties of modeled SM donors, including FMO energy level, ESP, dipole moment, and molecular planarity. The figures and energy levels of FMOs are shown in SI Fig. S2. As expected, the HOMO and LUMO energies of fluorinated SM donors are downshifted and energy gaps $\left(E_{\mathrm{g}} \mathrm{s}\right)$ are slightly widened with respect to prototype $0 \mathrm{~F}$. The decrease of HOMO energy can improve the $V_{\mathrm{OC}}$ of $\mathrm{OSC}$, but may lessen the driving force for hole transfer at donor-acceptor interface. Therefore, it is hard to make a judgement for the change of performance of the OSCs based on these fluorinated donors by simply comparing FMOs.

Next, the ESP distributions on the van del Waal surfaces are presented in Fig. 2a since they are essential to explore the molecular stacking in amorphous forms. For OF, the ESP on three BDT-T units is slightly negative, and the range is around $-8 \sim 0$ $\mathrm{kcal} / \mathrm{mol}$. Besides, the highest ESP is located at the joint area between side BDT-T and terminal acceptor unit, and is up to $18.3 \mathrm{kcal} / \mathrm{mol}$. In contrast, the ESP minimum $(-29.9 \mathrm{kcal} / \mathrm{mol})$ locates on one nitrogen atom of terminal acceptor unit. For fluorinated molecules, because of the strong electronegativity of fluorine atom, the fluorination on the lateral-chain thiophene can increase the ESP of entire molecule. Hence, the surface area with positive ESP increases with more fluorinations (see Fig. 2b). Overall, the averaged ESP of all fluorinated molecules has following order: $1 \mathrm{~F}$-all $>2 \mathrm{~F}$-all $>1 \mathrm{~F}$-side $>2 \mathrm{~F}$-side $>2 \mathrm{~F}$-center $>1 \mathrm{~F}$-center $>0 \mathrm{~F}$. Due to the small atomic radius of fluorine and its lone pair electrons, it seems that more fluorinations (from 1F- to 2F-substitutions) do not always increase the averaged ESP.

For center-fluorinated molecules (1F-center and 2F-center), all $\mathrm{F}$ atoms locate on the same side of molecule plane (see Fig. 2a). However, for the side-fluorinated molecules (1F-side and 2F-side), the $\mathrm{F}$ atoms locate on both sides (the front and 
reverse sides) equally. These different orientations of lateral-chain thiophenes after fluorinations have effects on dipole moments. The differences between the numbers of $\mathrm{F}$ atoms on the front and the reverse sides of molecular planes are $0 / 2 / 0 / 2 / 4 / 0 / 4$ for 0F/1F-center/1F-side/1F-all/2F-center/2F-side/2F-all, respectively. These unbalanced distributions of $\mathrm{F}$ atoms on two sides of molecular planes would increase the dipole moments and molecular backbone bending. Indeed, as shown in Fig. 2c, the dipole moments of $0 \mathrm{~F}, 1 \mathrm{~F}$-side, and 2F-side with balanced fluorinations are quite small, and they are $0.33,0.47$, and $0.55 \mathrm{D}$ respectively. Generally, the dipole moments of 2F-substituted molecules are about twice those of a 1F-substituted molecule, except for $1 \mathrm{~F}$-side and $2 \mathrm{~F}$-side which have balanced $\mathrm{F}$ distributions. Besides, due to the unbalanced distribution of $\mathrm{F}$ atoms and different numbers and positions of $\mathrm{F}$ substitutions, the backbone bending of the center-fluorinated molecules is stronger than that of the side- or all-fluorinated molecules, as shown in Fig. 2c (the estimation method of backbone bending is given in SI Fig. S3).

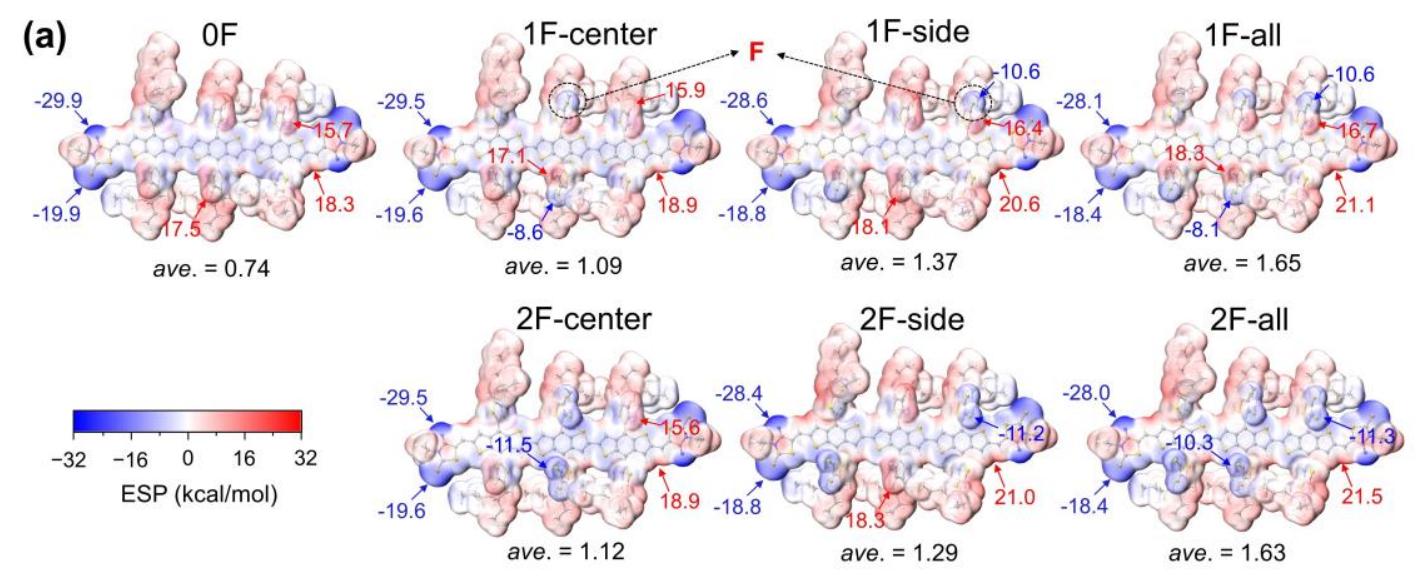

(b)

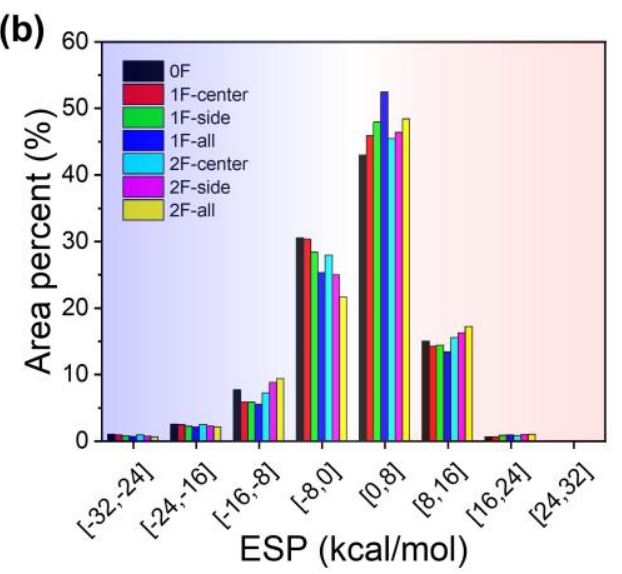

(c)

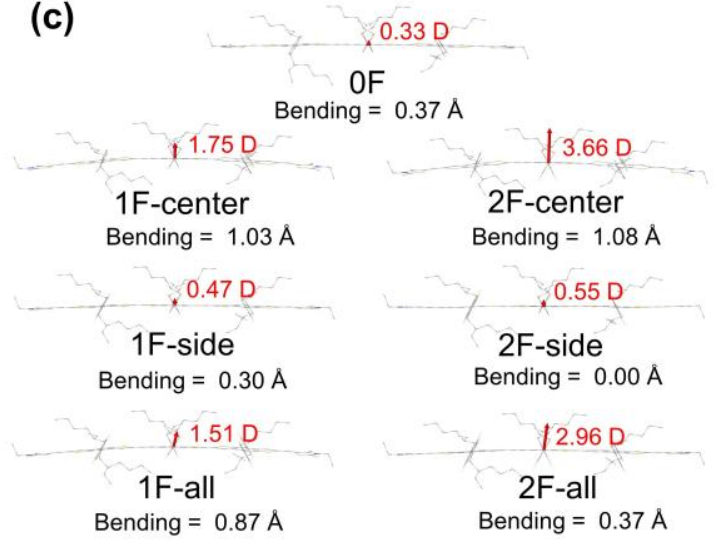


Fig. 2 (a) Electrostatic potential (ESP) maps of seven modeled molecules. (b) The percent of area with ESP in a specific region. (c) Dipole moments and the estimated backbone bending of seven molecules.

\subsection{Molecular packing in amorphous films}

Now we switch to the results of molecular dynamics simulations. Firstly, the radial distribution function (RDF) of the COM of donor unit (three BDT-T units only, excluding alkyl chains) are given in Fig. 3a. The sharp peaks of donor-donor RDFs at ca. $\sim 0.54-0.58 \mathrm{~nm}$ denote the parallel backbone $\pi-\pi$ stacking along molecular long axes with the inter-planar distance at $\sim 0.36-0.42 \mathrm{~nm}$. This is in good agreement with experimental result of $0 \mathrm{~F}(0.36 \mathrm{~nm})$ based on grazing-incidence small-angle X-ray scattering (GIWAXS) measurements. ${ }^{34}$ On the other hand, the values $(<1.5)$ of RDFs of ending groups (see SI Fig. S4) are much smaller than those of donor units, indicating that the acceptor-acceptor stacking is not dominant in amorphous forms. This finding is different from the previous MD simulation results of the A-D-A type of FREA ITIC, in which the terminal A-A stacking is dominant. ${ }^{35}$
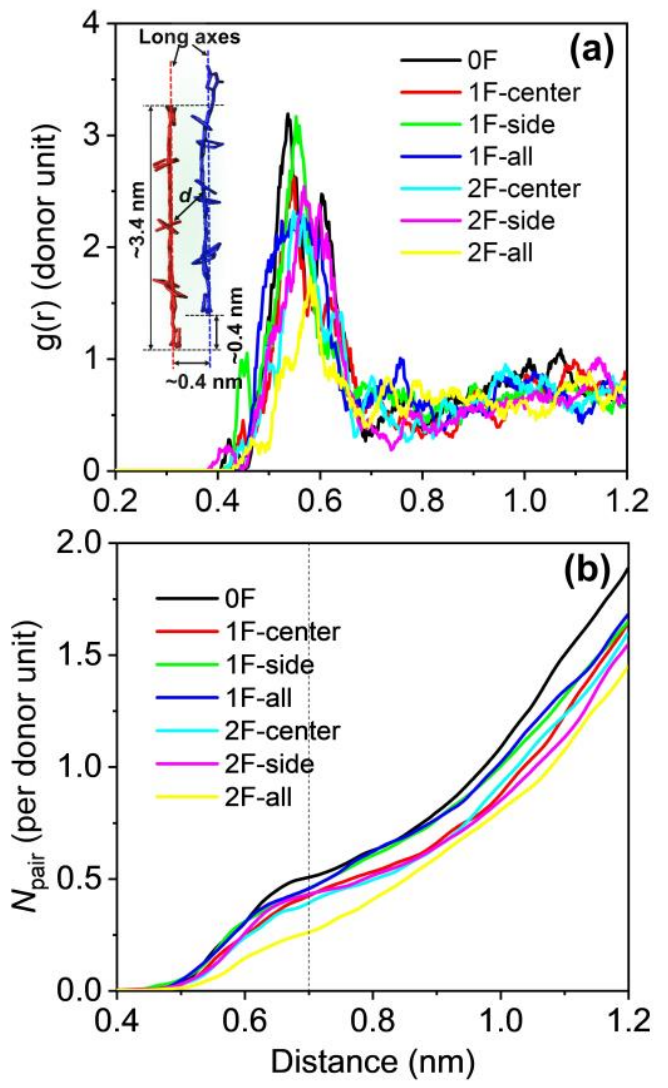
Fig. 3 (a) COM RDFs of donor units (three-BDT-T unit without alkyl chains) of seven molecules in amorphous films. (b) The number of pairs per donor unit as a function of donor-donor COM distance.

In Fig. 3a, OF shows the strongest RDF peak at $0.54 \mathrm{~nm}$ with a shoulder peak at $0.60 \mathrm{~nm}$, demonstrating the best crystallization among all simulated molecules. Besides, 1F-side also shows the second strongest RDF peak at $0.56 \mathrm{~nm}$, but without obvious shoulder peak. Though the RDF peak of $1 \mathrm{~F}$-all is not so strong as both of $0 \mathrm{~F}$ and $1 F$-side, its larger RDF value between 0.45 and $0.50 \mathrm{~nm}$ demonstrates that its crystallization is not bad too. Compared to other molecules, the RDF peak of $2 \mathrm{~F}$-all is clearly lower than others. In addition, the numbers of pairs ( $\left.N_{\text {pairs }}\right)$ of donor units as the function of the COM distance between two donor parts of two adjacent molecules are shown in Fig. 3b. When the distance cut-off is about $0.70 \mathrm{~nm}$ (the first valley of RDFs), the $N_{\text {pairs }}$ of all fluorinated molecules are smaller than that of $0 \mathrm{~F}$. Hence, we can conclude that the crystallinity of DRTB-T is weakened by fluorination. For $1 \mathrm{~F}$ substitutions, the $N_{\text {pair }}$ of $1 \mathrm{~F}$-all is similar to that of $1 \mathrm{~F}$-side, and $1 \mathrm{~F}$-center shows the smallest $N_{\text {pair }}$ among $1 \mathrm{~F}$-substituted molecules, implying its worst crystallization among all 1F-substituted molecules. For $2 \mathrm{~F}$ substitutions, the trend of $N_{\text {pairs }}$ is different, and the order is $2 \mathrm{~F}$-side $>2 \mathrm{~F}$-center $>2 \mathrm{~F}$-all. Notably, for the same substitution positions, the $N_{\text {pairs }}$ of $2 \mathrm{~F}$-substituted molecules are smaller than those of 1 F-substituted molecules, indicating worse crystallinity with more fluorinations. It can be attributed to the repulsion between negative charges on $\mathrm{F}$ atoms (see Figure 2) and the increased torsion angles (SI Fig. S5) between lateral-chain thiophenes and BDTs in 2F-substituted molecules, which can greatly impede the backbone $\pi-\pi$ stacking. As shown in SI Fig. S5, for $0 \mathrm{~F}$ and the 1F-substituted molecules, the dihedral angles between lateral-chain thiophene and BDT unit mainly distribute at around $57^{\circ}$. But for $2 \mathrm{~F}$-substituted molecules, they increase to around $65^{\circ}$ due to the coulomb repulsion between fluorine and sulfur atoms. Obviously, functionalizing the lateral-chain thiophene of BDT-T with two F atoms would enlarge the torsion angles between lateral-chain thiophene and BDT. Furthermore, the $N_{\text {pairs }}$ of $1 \mathrm{~F}$-center/2F-center are smaller than those of $1 \mathrm{~F}$-side/2F-side, respectively. This can be attributed to the unbalanced ESP and large backbone bending of center-substituted 
molecules (see Fig. 2), which hinder the backbone $\pi-\pi$ stacking between adjacent molecules.

(a)

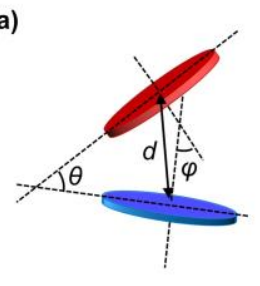

(b)

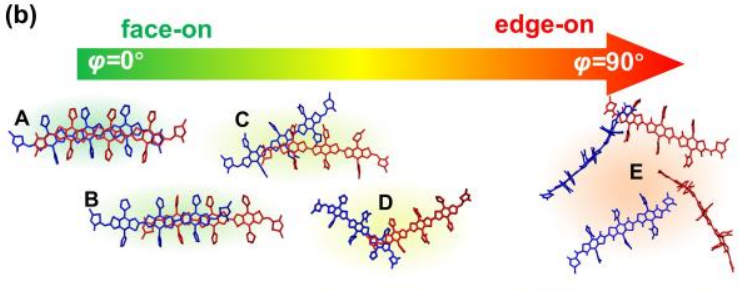

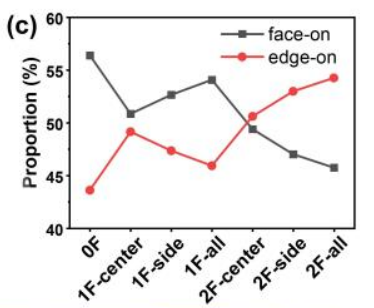
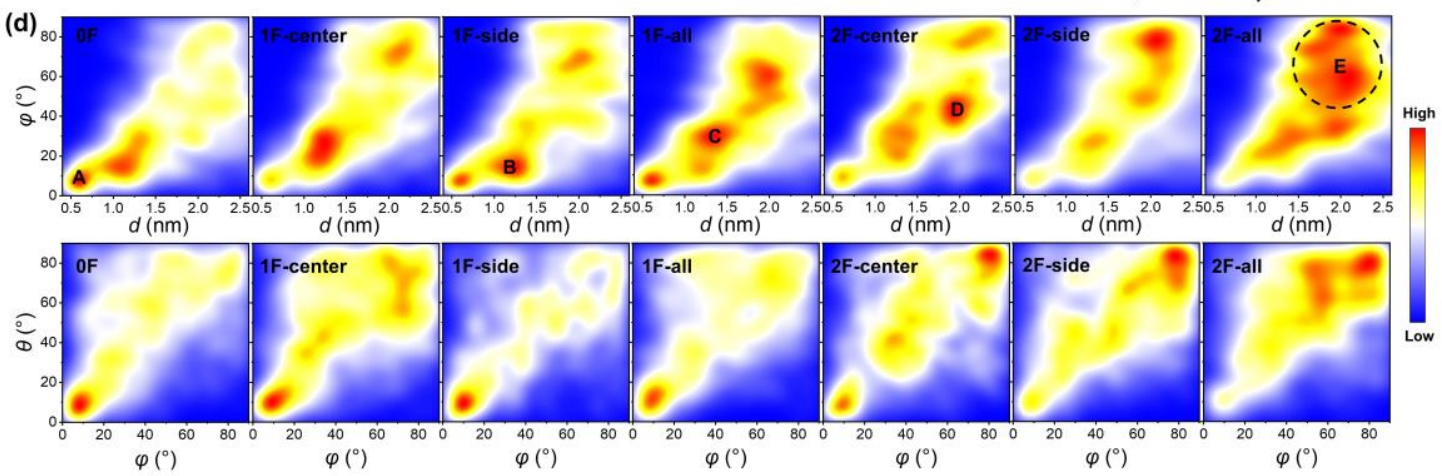

Fig. 4 (a) Scheme of three selected geometry parameters of dimer configurations: the backbone COM distance: $d$, the dihedral angle between two molecular planes: $\varphi$, and the angle between long axes: $\theta$. (b) Illustration of five representative configurations: A, B, C, D, and E. Note that the side alkyl chains and hydrogen atoms are omited for clarity. (c) Proportions of face-on and edge-on dimer configurations in the final MD boxes. (d) Density contour maps of $d-\varphi$ and $\varphi-\theta$ of molecular pairs in the final MD boxes. The two molecules with the shortest atomic distance between the atoms of two backbones smaller than $0.5 \mathrm{~nm}$ are defined as a pair.

To obtain more detailed analyses for amorphous forms studied in the present work, we characterize the dimer configurations formed by two neighboring molecules with three parameters: the COM distance between two molecular backbones $(d)$, the dihedral angle between two molecular planes $(\varphi)$, and the angle between the molecular long axes $(\theta)$, as schematized in Fig. 4a. The density contour maps of $d-\varphi$ and $\varphi-\theta$ in seven final MD boxes are presented in Fig. $4 \mathrm{~d}$. Note that we do not draw the density for $d>2.5 \mathrm{~nm}$ because it is very low. By carefully analyzing the $d, \varphi$, and $\theta$ of all molecular pairs (the bimolecular systems with the shortest atomic distances between two molecular backbones less than $0.5 \mathrm{~nm}$ are defined as pairs) in the final simulated box, ${ }^{37}$ we find five representative types of dimer configurations. The structures and classifications of these configurations A E are given in Fig. $4 b$. The typical configuration $\mathrm{A}$ ( $d$ around $0.6 \mathrm{~nm}$ and both $\theta$ and $\varphi<20^{\circ}$ ) generally has the 
strongest parallel $\pi-\pi$ stacking between backbones along long axes. The configuration $\mathrm{B}\left(d\right.$ around $1.2 \mathrm{~nm}$ and both $\theta$ and $\varphi<20^{\circ}$ ) has less intermolecular overlapping along the long axes than the configuration A. For configurations $\mathrm{C}$ and $\mathrm{D}$, its $\theta$ and $\varphi$ are larger $\left(>20^{\circ}\right.$ but $\left.<45^{\circ}\right)$ so that the $d(1.3-2.1 \mathrm{~nm})$ is longer than those in A and $\mathrm{B}$ configuration. The $\theta$ and $\varphi$ of configuration $\mathrm{E}$ are larger than $60^{\circ}$ and the $d$ is longer than $1.5 \mathrm{~nm}$. Overall, according to the value of $\varphi$, the dimers can be roughly classified into face-on $\left(\varphi<45^{\circ}\right)$ and edge-on $\left(\varphi>45^{\circ}\right)$ configurations. Therefore, we approximately classify the molecular pairs A, B, C and D as face-on configurations, and $\mathrm{E}$ as edge-on configuration.

Clearly, the density of configurations A, B, and C is much higher than that of D and $\mathrm{E}$ in $\mathrm{OF}$ final box because of its smallest average ESP and its small bending (see Fig. 2). This is consistent with the RDF analyses. For 1F substitutions, the density of configuration $\mathrm{A}$ in $1 \mathrm{~F}$-side and $1 \mathrm{~F}$-all boxes are similar to that of $0 \mathrm{~F}$, while the density of configuration $\mathrm{A}$ in $1 \mathrm{~F}$-center box is much smaller. Besides, the density of configuration $\mathrm{E}$ with both $\theta$ and $\varphi>60^{\circ}$ in $1 \mathrm{~F}$-center box is much higher than that in the other two boxes. This can be attributed to the unbalanced distribution of $\mathrm{F}$ atoms on BDT-T core and the large backbone bending in 1F-center molecule, as discussed above. The density of configurations $\mathrm{A}, \mathrm{B}$, and $\mathrm{C}$ in $1 \mathrm{~F}$-all box is comparable to that in $0 \mathrm{~F}$ box. However, different from $0 \mathrm{~F}$, in $1 \mathrm{~F}$-all box, there also shows the high density of configurations D and E because of its increased ESPs and large bending (see Fig. 2). Overall, we can see that the face-on configurations increases with more fluorinations in $1 \mathrm{~F}$ substituted boxes, as shown in Fig. 4c. For the dimer configurations in the $2 \mathrm{~F}$-substituted boxes, the situation is quite different from the 1F-substituted boxes. The density of configurations A, B, and C in 2F-substituted boxes is clearly smaller than that in $0 \mathrm{~F}$ and $1 \mathrm{~F}$-substituted boxes, and high density spots move to the upper right corner. This can be attributed to the coulomb repulsion between negative charges on two $\mathrm{F}$ atoms and the enlarged torsion angles (SI Fig. S5) between lateral-chain thiophenes and BDTs in the 2F-substituted molecules, which can greatly hinder the backbone $\pi-\pi$ stacking. Moreover, compared to $0 \mathrm{~F}$ and the $1 \mathrm{~F}$-substitutied boxes, the 2F-substituted boxes have much more edge-on 
configurations. This is reasonable because the face-on configurations are impeded by the repulsion between negative ESPs on F atoms and the large torsion angles (see SI Fig. S4) between lateral-chain thiophene and BDT in 2F-substituted molecules. And the decrease of face-on configurations is in turn beneficial to the edge-on configurations. Generally, the face-on configurations decreases with more fluorinations in $2 \mathrm{~F}$-substituted boxes.

The proportions of face-on and edge-on configurations are shown in Fig. 4c. As expected, compared to $0 \mathrm{~F}$ box, the proportions of face-on configurations in all boxes with fluorinated molecules are lowered by fluorination. Specifically, the face-on proportion in $0 \mathrm{~F}$ box is up to $56.4 \%$. In contrast, the proportions of face-on configurations in $1 \mathrm{~F}$-center/1F-side/ $1 \mathrm{~F}$-all/2F-center/2F-side/2F-all boxes are $50.8 \% / 52.6 \% / 54.1 \% / 49.4 \% / 47.0 \% / 45.7 \%$, respectively. Hence, the trend of face-on proportions is $1 \mathrm{~F}$-all $>1 \mathrm{~F}$-side $>1 \mathrm{~F}$-center in the $1 \mathrm{~F}$-substituted boxes, whereas the trend is $2 \mathrm{~F}$-all $<2 \mathrm{~F}$-side $<2 \mathrm{~F}$-center in $2 \mathrm{~F}$-substituted boxes. Interestingly, there are $1738 / 1766 / 1721 / 1744 / 1814 / 1863 / 1928$ counted molecule pairs in the simulated box of $0 \mathrm{~F} / 1 \mathrm{~F}$-center/1F-side/1F-all/2F-center/2F-side/2F-all respectively. This means that the edge-on molecular packing generally can improve the coordination numbers of these molecules, though it is harmful to $\pi-\pi$ stacking.

\subsection{Electronic coupling, reorganization energy, and hole mobility}

The charge transport of organic simiconductors in amorphous forms is determined by the mesoscopic electrical networks, which depend on the number of nearest neighbors and the strength of intermolecular electronic couplings $\left(V_{\mathrm{ij}}\right) .{ }^{63}$ The averaged electronic connectivity $\left(N_{\mathrm{c}}\right)$ per molecule, which is defined as the average number of neighbors for each molecule with $V_{\mathrm{ij}}$ larger than a given threshold $V_{\mathrm{T}}$, is helpful to study the strength of electrical networks. ${ }^{36}$ For a given $V_{\mathrm{T}}$, the $N_{\mathrm{c}}$ per molecule can be calculated by: $N_{\mathrm{c}}\left(V_{\mathrm{T}}\right)=N_{\text {pair }}\left(V_{\mathrm{ij}}>V_{\mathrm{T}}\right) \times 2 / N$, where $N_{\text {pair }}\left(V_{\mathrm{ij}}>V_{\mathrm{T}}\right)$ is the number of molecule pairs with $V_{\mathrm{ij}}>V_{\mathrm{T}}$, and $N$ is the number of molecules in the simulated box. Generally, a continuous and robust electrical network can be formed only when each molecule has over 2 connected neighbors, i.e. $N_{\mathrm{c}}>2 .{ }^{36}$ For seven 
final MD boxes in this work, the $N_{\mathrm{c}}$ per molecule is shown in Fig. 5a. As we can see, the $N_{\mathrm{c}}$ generally dcreases sharply with the increase of $V_{\mathrm{T}}$. When $V_{\mathrm{T}}=1 \mathrm{meV}$, the $N_{\mathrm{c}}$ of $0 \mathrm{~F} / 1 \mathrm{~F}$-center/1F-side/1F-all/2F-center/2F-side/2F-all

2.38/1.96/2.46/2.49/2.27/2.30/2.05, respectively. Besides, when $N_{\mathrm{c}}=2$, the $V_{\mathrm{TS}}$ in all boxes are smaller than $2 \mathrm{meV}$, indicating that the electrical networks are mainly formed by moleculear pairs with $V_{\mathrm{ij}}$ around $1 \mathrm{meV}$. In general, $1 \mathrm{~F}$-all and $1 \mathrm{~F}$-side show the strongest and the second strongest electrical networks respectively, while 1 F-center shows the smallest $N_{\mathrm{c}}$ among all molecules. In addition, when $V_{\mathrm{T}}<20 \mathrm{meV}$, the $N_{\mathrm{c}}$ of $2 \mathrm{~F}$-side is clearly smaller than those of $0 \mathrm{~F}, 1 \mathrm{~F}$-side, and $2 \mathrm{~F}$-center. However, when $V_{\mathrm{T}}>20 \mathrm{meV}$, the $N_{\mathrm{c}}$ of $2 \mathrm{~F}$-side is almost the same as those of $1 \mathrm{~F}$-side and $0 \mathrm{~F}$, and is clearly larger than those of $2 \mathrm{~F}$-center, $2 \mathrm{~F}$-all, and $1 \mathrm{~F}$-center.

The $V_{\mathrm{ij}}$ as the function of the backbone-backbone COM distance $d$ of molecular pairs extracted from the final MD boxes are shown in SI Fig. S6. Due to the disorder in amorphous forms, most of molecular pairs have small $V_{\mathrm{ij}} \mathrm{s}$ (less than $1 \mathrm{meV}$ ). These molecular pairs with $V_{\mathrm{ij}}<1 \mathrm{meV}$ contribute small to the continuous and robust electrical networks. To further explain the distinct differences of electrical networks among these final MD boxes, we analyze the number of pairs $\left(N_{\text {pair }}\right)$ with $V_{\mathrm{ij}}>1 \mathrm{meV}$ as the function of backbone COM distance $d$ or dihedral angel $\varphi$. As shown in Fig. 5b, in the regions $\mathrm{A}$ and $\mathrm{B}$, the trend of the $N_{\text {pairs }}$ is generally $0 \mathrm{~F}>1 \mathrm{~F}$-side $>1 \mathrm{~F}$-all $>$ $2 \mathrm{~F}$-side $>2 \mathrm{~F}$-center $>1 \mathrm{~F}$-center $>2 \mathrm{~F}$-all, which generally agree well with the RDF in Fig. 3. Among them, the $N_{\text {pair }}$ of 1 F-side increases sharply and becomes the largest in region $\mathrm{C}$, while the $N_{\text {pair }}$ of $1 \mathrm{~F}$-all becomes the largest in regions $\mathrm{D}$ and $\mathrm{E}$. As a result, the trend of the final $N_{\text {pair }}$ is $1 \mathrm{~F}$-all $>1 \mathrm{~F}$-side $>0 \mathrm{~F}>2 \mathrm{~F}$-center $>2 \mathrm{~F}$-side $>2 \mathrm{~F}$-all $>$ 1F-center. Notably, though 1F-center shows higher RDF peak than 2F-center, 2F-side, and 2F-all (see Fig. 3), it has the worst electrical network among all molecules. This is because of the strongest disorder (the areas with high values in the density contour map of Fig. 4d are far away to each other or not continuous) of the configurations in 1F-center box, which leads to bad electrical network. The $N_{\text {pairs }}$ with $V_{\mathrm{ij}}>1 \mathrm{meV}$ of $1 \mathrm{~F}$-center and $2 \mathrm{~F}$-all are always smaller than others; in the meantime, the $N_{\text {pairs }}$ with $V_{\mathrm{ij}}>1 \mathrm{meV}$ of $2 \mathrm{~F}$-side and $2 \mathrm{~F}$-center are quite close in all the range. For Fig. 5c, there 
are still many pairs with $V_{\mathrm{ij}}>1 \mathrm{meV}$ when $\varphi$ is in the range of $50^{\circ} \sim 90^{\circ}$, indicating configuration E also contributes to electrical networks. As expected, most of $N_{\text {pair }}$ with $V_{\mathrm{ij}}>1 \mathrm{meV}$ are contributed from face-on configurations.
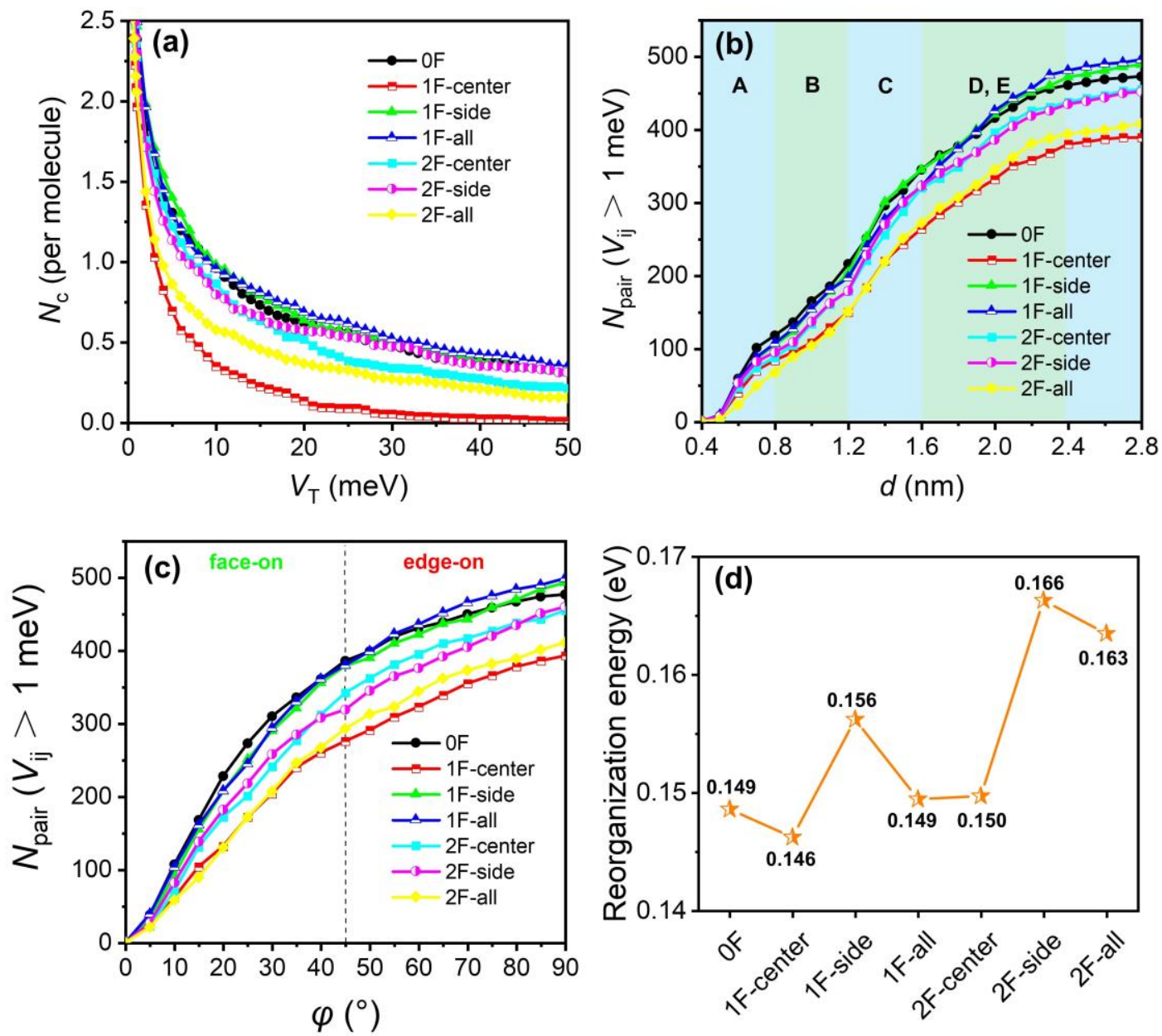

Fig. 5 (a) The averaged electronic connectivity per molecule as a function of $V_{\mathrm{T}}$ in seven final MD boxes. (b) The number of pairs with $V_{i j}>1 \mathrm{meV}$ as the function of backbone COM distance. (c) The number of pairs with $V_{i j}>1 \mathrm{meV}$ as the function of dihedral angle $\varphi$. (d)The inner reorganization energies of seven molecules.

Next, the reorganization energy $\lambda$ for hole transfer of seven monomers is given in Fig. $5 \mathrm{~d}$. The $\lambda$ of $0 \mathrm{~F}$ is $0.149 \mathrm{eV}$, and the $\lambda$ of the fluorinated molecules are generally not less than $0 \mathrm{~F}$ except for $1 \mathrm{~F}$-center $(0.146 \mathrm{eV})$. The trends of $\lambda$ of $1 \mathrm{~F}$ - and 2F-substituted molecules are always center $<$ all $<$ side. Also, for the same substitution position, the $\lambda$ of the $2 \mathrm{~F}$-substituted molecules is always larger than that of the 1F-substituted molecules. The differences among these $\lambda$ can be explained with the HOMO distribution, as shown in SI Fig. S2. Generally, the extended delocalization of FMOs can attenuate the inner reorganization energy. ${ }^{50,64}$ For 1 F-side 
and 2F-side, the HOMOs are mainly localized on the center BDT, while both of $1 \mathrm{~F}$-center and 2F-center are well delocalized on three BDTs. Hence, the $\lambda$ of $1 \mathrm{~F}$-side $(0.156 \mathrm{eV}) / 2 \mathrm{~F}$-side $(0.166 \mathrm{eV})$ are slightly larger than that of $1 \mathrm{~F}$-center $(0.146 \mathrm{eV}) /$ $2 \mathrm{~F}$-center $(0.150 \mathrm{eV})$, respectively. In addition, the HOMO delocalization of $2 \mathrm{~F}$-substituted molecules is worse than that of $1 \mathrm{~F}$-substituted molecules.

Finally, the hole mobilities $\left(\mu_{h}\right)$ are presented in Fig. 6. The calculated $\mu_{h}$ of prototype $0 \mathrm{~F}$ is $1.89 \times 10^{-3} \mathrm{~cm}^{2} \mathrm{~V}^{-1} \mathrm{~s}^{-1}$, which is one magnitude larger than the experiment result measured by the SCLC method $\left(1.88 \times 10^{-4} \mathrm{~cm}^{2} \mathrm{~V}^{-1} \mathrm{~s}^{-1}\right){ }^{34}$ It is acceptable because the experimental thin films were obtained by solvent vapor annealing (SVA). The quasi-ordered structure in the experimental work is harmful to form continuous electrical networks because there are many gaps between ordered regions, as reported by previous theoretical results. ${ }^{36}$ Compared to $0 \mathrm{~F}$, because of the strongest and second strongest electrical networks and different reorganization energies $\left(1 \mathrm{~F}\right.$-all $<1 \mathrm{~F}$-side), $1 \mathrm{~F}$-all $\left(3.19 \times 10^{-3} \mathrm{~cm}^{2} \mathrm{~V}^{-1} \mathrm{~s}^{-1}\right)$ and $1 \mathrm{~F}$-side $\left(3.05 \times 10^{-3} \mathrm{~cm}^{2}\right.$ $\mathrm{V}^{-1} \mathrm{~s}^{-1}$ ) show the fastest and the second fastest $\mu_{h} \mathrm{~s}$, respectively. Overall, the trend of $\mu_{h} \mathrm{~s}$ is $1 \mathrm{~F}$-center $<1 \mathrm{~F}$-side $<1 \mathrm{~F}$-all in the $1 \mathrm{~F}$-substituted molecules, whereas it is $2 \mathrm{~F}$-center $>2 \mathrm{~F}$-side $>2 \mathrm{~F}$-center in the $2 \mathrm{~F}$-substituted molecules. This is in good agreement with the proportions of face-on configurations (see Fig. 3c). It is reasonable because it is well known that the face-on configuration is beneficial to large $V_{\mathrm{ij}}$. Notably, the crystallinity and electrical network of $2 \mathrm{~F}$-center are weaker than both of $0 \mathrm{~F}$, but the $\mu_{h}$ of the $2 \mathrm{~F}$-center $\left(2.23 \times 10^{-3} \mathrm{~cm}^{2} \mathrm{~V}^{-1} \mathrm{~s}^{-1}\right)$ is larger than that of $0 \mathrm{~F}$. It can be attributed to that the number of configurations $\mathrm{C}$ and $\mathrm{D}$ of $2 \mathrm{~F}$-center is much larger (see Fig. 4d) than that of 0F. In general, a short charge transfer distance is beneficial for enhancing electronic coupling, while a large charge transfer distance is favorable for large carrier mobility, as shown in Eqn (2). Thus, achieving large charge carrier mobility should ensure enhanced electronic coupling and long charge transfer distance simultaneously. ${ }^{65}$ Though the $V_{\mathrm{ij}} \mathrm{s}$ of configurations $\mathrm{C}$ and $\mathrm{D}$ may be smaller than those of configurations $\mathrm{A}$ and $\mathrm{B}$, they can provide longer charge transfer pathways. So, the configurations $\mathrm{C}$ and $\mathrm{D}$ are also important to improve $\mu_{h}$. In contrast, though the electrical network of $2 \mathrm{~F}$-side is comparable to $2 \mathrm{~F}$-center when $V_{\mathrm{T}}$ 
$<20 \mathrm{meV}$, and even is better than that of $2 \mathrm{~F}$-center when $V_{\mathrm{T}}>20 \mathrm{meV}$, the $\mu_{h}$ of $2 \mathrm{~F}$-side $\left(1.65 \times 10^{-3} \mathrm{~cm}^{2} \mathrm{~V}^{-1} \mathrm{~s}^{-1}\right)$ is slightly smaller than both of $0 \mathrm{~F}$ and $2 \mathrm{~F}$-center. This is because $2 \mathrm{~F}$-side has less configurations $\mathrm{C}$ and $\mathrm{D}$ as well as larger reorganization energy than 2F-center. Similarly, though the electronic coupling of 2F-all is slightly stronger than that of $1 \mathrm{~F}$-center, the $\lambda$ of $2 \mathrm{~F}$-all $(0.163 \mathrm{eV})$ is larger than that of $1 \mathrm{~F}$-center $(0.146 \mathrm{eV})$, hence, the $\mu_{h}$ of $2 \mathrm{~F}$-all $\left(0.95 \times 10^{-3} \mathrm{~cm}^{2} \mathrm{~V}^{-1} \mathrm{~s}^{-1}\right)$ is slightly smaller than $1 \mathrm{~F}$-center $\left(1.15 \times 10^{-3} \mathrm{~cm}^{2} \mathrm{~V}^{-1} \mathrm{~s}^{-1}\right)$. Furthermore, due to the much weaker electrical networks of $1 \mathrm{~F}$-center and $2 \mathrm{~F}$-all, their $\mu_{h} \mathrm{~s}$ are smaller than that of prototype $0 \mathrm{~F}$.

We also note that recent reports show the $\mu_{h}$ of $0 \mathrm{~F}: \mathrm{F}-2 \mathrm{Cl}$ blend is $2.50 \times 10^{-4} \mathrm{~cm}^{2}$ $\mathrm{V}^{-1} \mathrm{~s}^{-1}$, and it is clearly larger than that of $1 \mathrm{~F}$-center:F-2Cl blend $\left(8.56 \times 10^{-5} \mathrm{~cm}^{2} \mathrm{~V}^{-1}\right.$ $\left.\mathrm{s}^{-1}\right) .{ }^{32,33}$ This trend is consistent with our result that the $\mu_{h}$ of $0 \mathrm{~F}\left(1.89 \times 10^{-3} \mathrm{~cm}^{2} \mathrm{~V}^{-1}\right.$ $\left.\mathrm{s}^{-1}\right)$ is faster than that of $1 \mathrm{~F}$-center $\left(1.15 \times 10^{-3} \mathrm{~cm}^{2} \mathrm{~V}^{-1} \mathrm{~s}^{-1}\right)$. Besides, our results show that the $\mu_{h}$ of $2 \mathrm{~F}$-center $\left(2.23 \times 10^{-3} \mathrm{~cm}^{2} \mathrm{~V}^{-1} \mathrm{~s}^{-1}\right)$ is superior to both of $0 \mathrm{~F}$ and $1 \mathrm{~F}$-center. It is also in good agreement with the previous experimental results that BTEC-2F:Y6 blend exhibited a higher hole mobility of $5.43 \times 10^{-4} \mathrm{~cm}^{2} \mathrm{~V}^{-1} \mathrm{~s}^{-1}$ than prototype DCAO3TBDTT:Y6 $\left(4.60 \times 10^{-4} \mathrm{~cm}^{2} \mathrm{~V}^{-1} \mathrm{~s}^{-1}\right)$ and BTEC-1F:Y6 $\left(4.17 \times 10^{-4}\right.$ $\mathrm{cm}^{2} \mathrm{~V}^{-1} \mathrm{~s}^{-1}$ ) (BTEC-1F and BTEC-2F are obtained by functionalizing the lateral-thiophenes of the center BDT-T unit of DCAO3TBDTT with 1 and $2 \mathrm{~F}$ atoms, respectively) by Ge et al. ${ }^{18}$ In brief, the available experimental results confirm the reliability of our methods to predict of trends of hole mobility of fluorinated BDT-T based donor materials. 


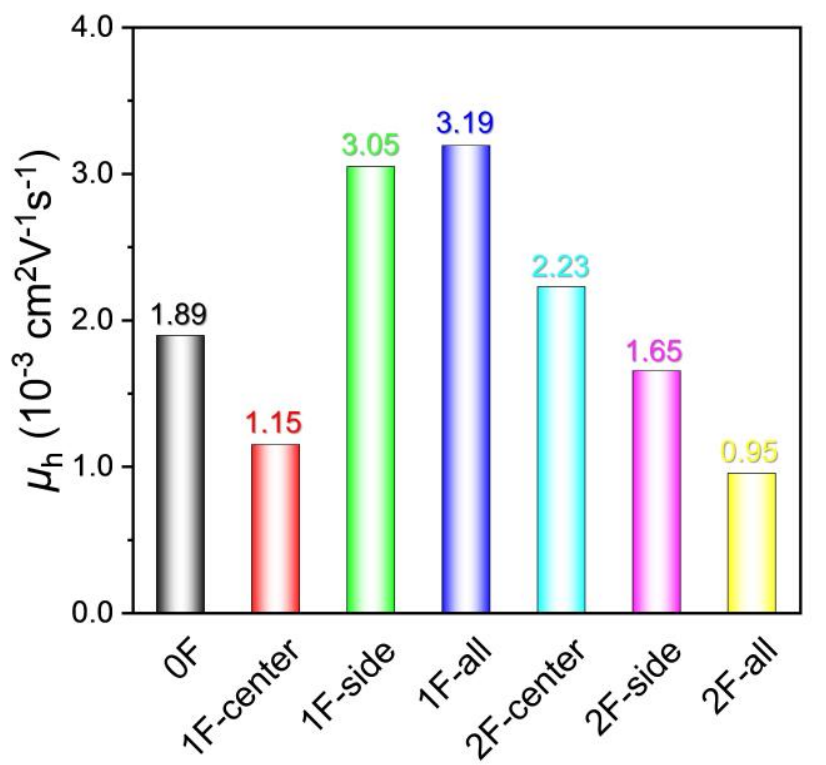

Fig.6 Hole mobilities $\left(\mu_{h}\right)$ of seven simulated molecules, obtained with molecular dynamics simulation and KMC.

\section{Conclusions}

In summary, we systematically studied the effects of the numbers and positions of fluorinations on the morphology and hole mobility of DRTB-T donor by using multiscale simulations. We find that the crystallinity and face-on configurations of DRTB-T (0F) are weakened by fluorination, and the molecular stacking patterns also change significantly by the number and position of fluorination. The trend of face-on configurations proportions in the $1 \mathrm{~F}$-substituted molecules is $1 \mathrm{~F}$-center $<1 \mathrm{~F}$-side $<$ $1 \mathrm{~F}$-all, whereas it is $2 \mathrm{~F}$-center $>2 \mathrm{~F}$-side $>2 \mathrm{~F}$-all in the $2 \mathrm{~F}$-substituted molecules. The face-on configurations are dominated in $0 \mathrm{~F}$ and $1 \mathrm{~F}$-substituted molecules. However, the edge-on configurations are dominated in $2 \mathrm{~F}$-substituted molecules because of the repulsion between the negative ESP on F atoms and the large torsion angles between lateral-chain thiophene and BDTs, which greatly impede the backbone $\pi-\pi$ stacking. Besides, due to the changes of HOMO delocalization and molecule packing, the reorganization energy and electrical network also change greatly, respectively. As a result, the hole mobility greatly varies in different fluorinated donors, and the trend of obtained $\mu_{h}$ s match well with the experiments. We find that by carefully adjust the number and position of fluorination on the lateral-chain thiophenes of DRTB-T, 
because of the enhanced electrical networks and similar reorganization energies, the $\mu_{h} \mathrm{~s}$ of $1 \mathrm{~F}$-all $\left(3.19 \times 10^{-3} \mathrm{~cm}^{2} \mathrm{~V}^{-1} \mathrm{~s}^{-1}\right)$ and $1 \mathrm{~F}$-side $\left(3.05 \times 10^{-3} \mathrm{~cm}^{2} \mathrm{~V}^{-1} \mathrm{~s}^{-1}\right)$ are clearly faster to that of $0 \mathrm{~F}\left(1.89 \times 10^{-3} \mathrm{~cm}^{2} \mathrm{~V}^{-1} \mathrm{~s}^{-1}\right)$ and other fluorinated molecules. In contrast, due to the limited backbone $\pi-\pi$ stacking and weak electrical networks, the $\mu_{h} \mathrm{~s}$ of $1 \mathrm{~F}$-center $\left(1.15 \times 10^{-3} \mathrm{~cm}^{2} \mathrm{~V}^{-1} \mathrm{~s}^{-1}\right)$ and $2 \mathrm{~F}$-all $\left(0.95 \times 10^{-3} \mathrm{~cm}^{2} \mathrm{~V}^{-1} \mathrm{~s}^{-1}\right)$ are slower than that of $0 \mathrm{~F}$. Furthermore, the trend of $\mu_{h} \mathrm{~s}$ is $1 \mathrm{~F}$-center $<1 \mathrm{~F}$-side $<1 \mathrm{~F}$-all in the $1 \mathrm{~F}$-substituted molecules, whereas it is $2 \mathrm{~F}$-center $>2 \mathrm{~F}$-side $>2 \mathrm{~F}$-all in the 2F-substituted molecules. This is consistent with the proportions of face-on configurations. Finally, we find that functionalizing the lateral-chain thiophenes of side BDT-Ts (1F-side) or all BDT-Ts (1F-all) with only one $\mathrm{F}$ atom are efficient to fine-tune the morphologies and hole motilities of DRTB-T based SM donors at the same time.

\section{Conflicts of interest}

The author has no conflicts to declare.

\section{Acknowledgements}

The authors greatly thank for the financial support from Fundamental Research Funds for the Central Universities (XDJK2016A016, XDJK2016E014, XDJK2017D015, and XDJK2017D019), the Foundation for Returning Overseas Chinese (No. 102060-20600606), and the Start-up Funding of Young Faculty of Southwest University.

\section{References}

1 Q. Liu, Y. Jiang, K. Jin, J. Qin, J. Xu, W. Li, J. Xiong, J. Liu, Z. Xiao, K. Sun, S. Yang, X. Zhang and L. Ding, Sci. Bull., 2020, 65, 272-275.

2 C. Li, J. Zhou, J. Song, J. Xu, H. Zhang, X. Zhang, J. Guo, L. Zhu, D. Wei, G. Han, J. Min, Y. Zhang, Z. Xie, Y. Yi, H. Yan, F. Gao, F. Liu and Y. Sun, Nat. Energy, 2021, DOI: 10.1038/s41560-021-00820-x.

3 K. Jin, Z. Xiao and L. Ding, J. Semicond., 2021, 42, 010502.

4 J. Qin, L. Zhang, C. Zuo, Z. Xiao, Y. Yuan, S. Yang, F. Hao, M. Cheng, K. Sun, Q. Bao, Z. Bin, Z. Jin and L. Ding, J. Semicond., 2021, 42, 010501.

5 B. Kan, Y. Kan, L. Zuo, X. Shi and K. Gao, InfoMat, 2020, DOI: 10.1002/inf2.12163.

6 K. Sun, Z. Xiao, S. Lu, W. Zajaczkowski, W. Pisula, E. Hanssen, J. M. White, R. M. Williamson, J. Subbiah, J. Ouyang, A. B. Holmes, W. W. H. Wong and D. J. Jones, Nat. Commun., 2015, 6, 6013.

7 L. Yang, S. Zhang, C. He, J. Zhang, H. Yao, Y. Yang, Y. Zhang, W. Zhao and J. Hou, J. Am. 
Chem. Soc., 2017, 139, 1958-1966.

8 R. Zhou, Z. Jiang, C. Yang, J. Yu, J. Feng, M. A. Adil, D. Deng, W. Zou, J. Zhang, K. Lu, W. Ma, F. Gao and Z. Wei, Nat. Commun., 2019, 10, 5393.

9 A. Mishra and P. Bäuerle, Angew. Chem. Int. Ed., 2012, 51, 2020-2067.

10 C. An, Y. Qin, T. Zhang, Q. Lv, J. Qin, S. Zhang, C. He, H. Ade and J. Hou, J. Mater. Chem. A, 2021, 9, 13653-13660.

11 C. J. Brabec, S. Gowrisanker, J. J. M. Halls, D. Laird, S. Jia and S. P. Williams, $A d v$. Mater., 2010, 22, 3839-3856.

12 Y. He and Y. Li, Phys. Chem. Chem. Phys., 2011, 13, 1970-1983.

13 Y. Lin, J. Wang, Z. G. Zhang, H. Bai, Y. Li, D. Zhu and X. Zhan, Adv. Mater, 2015, 27, 1170-1174.

14 J. Yuan, Y. Zhang, L. Zhou, G. Zhang, H.-L. Yip, T.-K. Lau, X. Lu, C. Zhu, H. Peng, P. A. Johnson, M. Leclerc, Y. Cao, J. Ulanski, Y. Li and Y. Zou, Joule, 2019, 3, 1140-1151.

15 G. Zhang, J. Zhao, P. C. Y. Chow, K. Jiang, J. Zhang, Z. Zhu, J. Zhang, F. Huang and H. Yan, Chem. Rev., 2018, 118, 3447-3507.

16 J. Hou, O. Inganas, R. H. Friend and F. Gao, Nat. Mater, 2018, 17, 119-128.

17 Q. Yue, H. Wu, Z. Zhou, M. Zhang, F. Liu and X. Zhu, Adv. Mater, 2019, 31, 1904283.

18 J. Ge, L. Xie, R. Peng, B. Fanady, J. Huang, W. Song, T. Yan, W. Zhang and Z. Ge, Angew. Chem. Int. Ed., 2020, 59, 2808-2815.

19 J. Qin, C. An, J. Zhang, K. Ma, Y. Yang, T. Zhang, S. Li, K. Xian, Y. Cui, Y. Tang, W. Ma, H. Yao, S. Zhang, B. Xu, C. He and J. Hou, Sci. China Mater., 2020, DOI: 10.1007/s40843-020-1269-9.

20 H. Chen, D. Hu, Q. Yang, J. Gao, J. Fu, K. Yang, H. He, S. Chen, Z. Kan, T. Duan, C. Yang, J. Ouyang, Z. Xiao, K. Sun and S. Lu, Joule, 2019, 3, 3034-3047.

21 L. Yang, S. Zhang, C. He, J. Zhang, H. Yao, Y. Yang, Y. Zhang, W. Zhao and J. Hou, J. Am. Chem. Soc., 2017, 139, 1958-1966.

22 H. Zhang, H. Yao, J. Hou, J. Zhu, J. Zhang, W. Li, R. Yu, B. Gao, S. Zhang and J. Hou, Adv. Mater., 2018, 30, 1800613.

23 D. Hu, Q. Yang, Y. Zheng, H. Tang, S. Chung, R. Singh, J. Lv, J. Fu, Z. Kan, B. Qin, Q. Chen, Z. Liao, H. Chen, Z. Xiao, K. Sun and S. Lu, Adv. Sci., 2021, 8, 2004262.

24 M. Li, W. Ni, X. Wan, Q. Zhang, B. Kan and Y. Chen, J. Mater. Chem. A, 2015, 3, 4765-4776.

25 H. Yao, L. Ye, H. Zhang, S. Li, S. Zhang and J. Hou, Chem. Rev., 2016, 116, 7397-7457.

26 Y. Zhang, H. Yao, S. Zhang, Y. Qin, J. Zhang, L. Yang, W. Li, Z. Wei, F. Gao and J. Hou, Sci. China Chem., 2018, 61, 1328-1337.

27 M. Zhang, X. Guo, S. Zhang and J. Hou, Adv. Mater., 2014, 26, 1118-1123.

28 D. Deng, E. Zhou and Z. Wei, Acta Physico-Chim. Sin, 2018, 34, 1239-1249.

29 M. Zhang, X. Guo, W. Ma, H. Ade and J. Hou, Adv. Mater., 2015, 27, 4655-4660.

30 H. Wu, Q. Yue, Z. Zhou, S. Chen, D. Zhang, S. Xu, H. Zhou, C. Yang, H. Fan and X. Zhu, J. Mater. Chem. A, 2019, 7, 15944-15950.

31 X. Wang, J. Wang, J. Han, D. Huang, P. Wang, L. Zhou, C. Yang, X. Bao and R. Yang, Nano Energy, 2021, 81, 105612.

32 Y. Wang, Y. Wang, B. Kan, X. Ke, X. Wan, C. Li and Y. Chen, Adv. Energy Mater, 2018, 8, 1802021. 
33 C. Liu, N. Qiu, Y. Sun, X. Ke, H. Zhang, C. Li, X. Wan and Y. Chen, Front. Chem., 2020, $8,329$.

34 L. Yang, S. Zhang, C. He, J. Zhang, Y. Yang, J. Zhu, Y. Cui, W. Zhao, H. Zhang, Y. Zhang, Z. Wei and J. Hou, Chem. Mater., 2018, 30, 2129-2134.

35 G. Han, Y. Guo, X. Song, Y. Wang and Y. Yi, J. Mater. Chem. C, 2017, 5, 4852-4857.

36 G. Han, X. Shen, R. Duan, H. Geng and Y. Yi, J. Mater. Chem. C, 2016, 4, 4654-4661.

37 G. Han, Y. Guo, R. Duan, X. Shen and Y. Yi, J. Mater. Chem. A, 2017, 5, 9316-9321.

38 G. Han, X. Shen and Y. Yi, Adv. Mater. Interfaces, 2015, 2, 1500329.

39 W. M. E. Young, E. W., Proc. Phys. Soc., 1966, 89, 735.

40 L. Wang, Q. Li, Z. Shuai, L. Chen and Q. Shi, Phys. Chem. Chem. Phys., 2010, 12, 3309-3314.

41 M. J. Abraham, T. Murtola, R. Schulz, S. Páll, J. C. Smith, B. Hess and E. Lindahl, SoftwareX, 2015, 1-2, 19-25.

42 M. J. Frisch, G. W. Trucks, H. B. Schlegel, G. E. Scuseria, M. A. Robb, J. R. Cheeseman, G. Scalmani, V. Barone, B. Mennucci, G. A. Petersson, H. Nakatsuji, M. Caricato and X. H. Li, H. P.; Izmaylov, A. F.; Bloino, J.; Zheng, G.; Sonnenberg, J. L.; Hada, M.; Ehara, M.; Toyota, K.; Fukuda, R.; Hasegawa, J.; Ishida, M.; Nakajima, T.; Honda, Y.; Kitao, O.; Nakai, H.; Vreven, T.; Montgomery, J. A., Jr.; Peralta, J. E.; Ogliaro, F.; Bearpark, M.; Heyd, J. J.; Brothers, E.; Kudin, K. N.; Staroverov, V. N.; Kobayashi, R.; Normand, J.; Raghavachari, K.; Rendell, A.; Burant, J. C.; Iyengar, S. S.; Tomasi, J.; Cossi, M.; Rega, N.; Millam, N. J.; Klene, M.; Knox, J. E.; Cross, J. B.; Bakken, V.; Adamo, C.; Jaramillo, J.; Gomperts, R.; Stratmann, R. E.; Yazyev, O.; Austin, A. J.; Cammi, R.; Pomelli, C.; Ochterski, J. W.; Martin, R. L.; Morokuma, K.; Zakrzewski, V. G.; Voth, G. A.; Salvador, P.; Dannenberg, J. J.; Dapprich, S.; Daniels, A. D.; Farkas, Ö.; Foresman, J. B.; Ortiz, J. V.; Cioslowski, J.; Fox, D. J., Gaussian 2009 Revision D.01, Wallingford CT, 2009.

43 J. Wang, R. M. Wolf, J. W. Caldwell, P. A. Kollman and D. A. Case, J. Comput. Chem., 2004, 25, 1157-1174.

44 A. W. Sousa da Silva and W. F. Vranken, BMC Res. Notes, 2012, 5, 367.

45 C. A. Reynolds, J. W. Essex and W. G. Richards, J. Am. Chem. Soc., 1992, 114, 9075-9079.

46 T. Lu and F. Chen, J. Comput. Chem., 2012, 33, 580-592.

47 T. Lu and F. Chen, J. Mol. Graph. Model., 2012, 38, 314-323.

48 F. C. Tian Lu, Acta Chim. Sinica, 2011, 69, 2393-2406.

49 T. Hu, G. Han, Z. Tu, R. Duan and Y. Yi, J. Phys. Chem. C, 2018, 122, 27191-27197.

50 Z. Z. Sun, S. Feng, C. Gu, N. Cheng and J. Liu, Phys. Chem. Chem. Phys., 2019, 21, 15206-15214.

51 G. Bussi, D. Donadio and M. Parrinello, J. Chem. Phys., 2007, 126, 014101.

52 H. J. C. Berendsen, J. P. M. Postma, W. F. v. Gunsteren, A. DiNola and J. R. Haak, J. Chem. Phys., 1984, 81, 3684-3690.

53 W. G. Hoover, Phys. Rev. A, 1985, 31, 1695-1697.

54 S. Nosé, J. Chem. Phys., 1984, 81, 511-519.

55 A. R. M. Parrinello, J. Appl. Phys., 1981, 52, 7182-7190.

56 E. Gorham-Bergeron and D. Emin, Physical Review B, 1977, 15, 3667-3680.

57 V. Coropceanu, J. Cornil, D. A. da Silva Filho, Y. Olivier, R. Silbey and J. L. Brédas, 
Chem. Rev., 2007, 107, 926-952.

58 C. Yao, C. Peng, Y. Yang, L. Li, M. Bo and J. Wang, J. Mater. Chem. C, 2018, 6, $4912-4918$.

59 C. Yao, Y. Yang, L. Li, M. Bo, C. Peng and J. Wang, J. Mater. Chem. A, 2019, 7, 18150-18157.

60 R. A. Marcus, Rev. Mod. Phys., 1993, 65, 599-610.

61 J. Ridley and M. Zerner, Theor. Chim. Acta, 1973, 32, 111-134.

62 S. F. Nelsen, S. C. Blackstock and Y. Kim, J. Am. Chem. Soc., 1987, 109, 677-682.

63 B. M. Savoie, K. L. Kohlstedt, N. E. Jackson, L. X. Chen, M. Olvera de la Cruz, G. C. Schatz, T. J. Marks and M. A. Ratner, Proc. Natl. Acad. Sci. USA, 2014, 111, 10055.

64 T. Liu, K. Sun, R. He, Z. Zhang, W. Shen and M. Li, J. Phys. Chem. C, 2018, 122, 8804-8813.

65 C. Xu, X. Xu and S. Zheng, New J. Chem., 2020, 44, 15177-15185. 